\title{
Concepções de docentes em relação ao manual sobre violência intrafamiliar
}

\author{
Professors' opinions concerning a manual on \\ domestic violence
}

\author{
Edinilsa Ramos de Souza ${ }^{\mathrm{I}}$ \\ Ana Lúcia Ferreira ${ }^{I I}$ \\ Neuci Cunha dos Santos III
}

\author{
PALAVRAS-CHAVE \\ - Violência doméstica \\ - Materiais de ensino \\ - Formação de recursos humanos \\ - Educação de graduação \\ em medicina \\ - Programas de graduação \\ em enfermagem
}

\section{KEY WORDS}

- Domestic violence

- Teaching materials

- Human resources formation

- Education, medical, undergraduate

- Education, Nursing,

Diploma Programs
Aprovado em: 06/01/2009

\begin{abstract}
R ES U M O
Introdução: O Ministério da Saúde publicou o manual técnico Violência intrafamiliar: orientações para a prática em serviço como uma estratégia para identificar e intervir de forma adequada nas situações de violência intrafamiliar e preveni-las. Objetivos: Identificar percepções, críticas e sugestões de docentes da saúde sobre esse manual. Metodologia: Fez-se um estudo exploratório pautado na triangulação de métodos. Aplicou-se um questionário a uma amostra de conveniência de docentes de faculdades de Medicina e de Enfermagem das cidades do Rio de Janeiro e Cuiabá, com questões abertas e fechadas sobre as características e aplicabilidade do manual. Resultados: O manual foi bem avaliado quanto às características e aplicabilidade. Contudo, a grande maioria dos docentes não o usa em suas aulas e considera seu conteúdo insuficiente para a formação do profissional em relação ao tema. Conclusão: Sugere-se usar este material em disciplinas oferecidas no início dos cursos da área da saúde, objetivando chamar a atenção dos graduandos para alguns aspectos epidemiológicos, clínicos e de prevenção da violência intrafamiliar, sem a pretensão de capacitá-los para o atendimento das vítimas.
\end{abstract}

\section{A B S T R A C T}

Introduction: The Brazilian Ministry of Health has published a technical manual entitled Domestic Violence: Guidelines for Health Services as a strategy to help identify, intervene in, and prevent domestic violence. Objectives: To identify insights, criticisms, and suggestions from professors of the various health fields concerning the manual. Methodology: An exploratory study was conducted, using the three-point method. A questionnaire was given to appropriate health field professors in the cities of Rio de Janeiro and Cuiabá, containing open-ended and closed questions on the manual's characteristics and practicality. Results: The manual's characteristics and practicality were both evaluated as good. However, most professors did not use the manual in their classes, finding its content insufficient for training health professionals. Conclusion: The manual is recommended for use in classes offered at the beginning of academic programs in the various health fields. The aim is to attract the attention of undergraduates in relation to epidemiological and clinical aspects and prevention of domestic violence, without intending to train them to treat victims.

\footnotetext{
${ }^{I}$ Fundação Oswaldo Cruz, Rio de Janeiro, Rio de Janeiro, Brasil.

${ }^{I I}$ Universidade Federal do Rio de Janeiro, Rio de Janeiro, Rio de Janeiro, Brasil.

II Universidade Federal de Mato Grosso, Cuiabá, Mato Grosso, Brasil.
} 


\section{INTRODUÇÃO}

A violência passou a ser considerada em nosso país como um problema de saúde pública e a destacar-se como um objeto de preocupação do setor apenas nas duas últimas décadas do século passado. É, portanto, um tema ainda em processo de inclusão lenta e gradual na agenda da saúde ${ }^{1}$.

Desde a década de 1990, o Ministério da Saúde vem elaborando diversas políticas, portarias, normatizações e manuais técnicos de orientação para a atuação do setor em relação à violência em todas as instâncias do SUS. Dentre esses instrumentos destaca-se a Política Nacional de Redução da Morbimortalidade por Acidentes e Violências (Portaria MS/GM no 737 de 16/05/2001), que faz um amplo diagnóstico da situação de violência no País e preconiza as principais diretrizes para atuação do setor saúde; a Portaria no 936/GM, de 2004, que dispõe sobre a estruturação da Rede Nacional de Prevenção da Violência e Promoção da Saúde e a Implantação e Implementação de Núcleos de Prevenção à Violência em Estados e Municípios²; o Pacto pela Saúde, que é um plano de ação nacional que aponta em uma de suas macroprioridades a prevenção e atenção aos acidentes e violências como um dos principais problemas de morbimortalidade $^{3}$; e o manual técnico Violência intrafamiliar: orientações para a prática em serviço ${ }^{4}$, que é um instrumento orientador para os profissionais da atenção básica em saúde.

Outros marcos e desdobramentos políticos importantes na prevenção e enfrentamento da violência intrafamiliar são as políticas específicas aos grupos populacionais da unidade familiar, tais como as voltadas para a mulher ${ }^{5,6}$, crianças e adolescentes ${ }^{7}$, idosos ${ }^{8}$ e portadores de deficiência ${ }^{9,10}$.

Tais propostas governamentais, além de outras desenvolvidas por órgãos representativos de entidades profissionais, vêm sendo fortalecidas com a implantação e implementação de orientações para as práticas em serviço voltadas para a prevenção, o monitoramento e o enfrentamento de situações de violência na família, principalmente por meio de manuais, relatórios e normas técnicas específicas para cada grupo ${ }^{11-17}$.

Apesar dos esforços empreendidos pelo Ministério da Saúde na formulação e implementação de políticas de prevenção e atenção às questões relacionadas à violência, ainda se verificam defasagens entre a criação e o desenvolvimento de programas e a capacitação de profissionais que atuam na rede SUS especificamente dirigidos a essa temática.

No que se refere à formação dos profissionais de saúde, a lacuna é ainda maior, pois os órgãos formadores, por um lado, ainda percebem muito pouco o tema como pertinente ao âmbito da saúde, desconhecem a sua magnitude e, consequentemente, não o priorizam como um problema a ser incorporado nos planejamentos curriculares de seus cursos. Por outro lado, parece não haver uma integração entre as políticas existentes e preconizadas pelo Ministério da Saúde quanto à formação dos profissionais da saúde e esses órgãos formadores.

Estudos têm reconhecido que os profissionais de saúde, pela posição que ocupam na prestação de serviços à população, são considerados essenciais na identificação de indivíduos e grupos populacionais de risco para a violência e para a execução de iniciativas que promovam a prevenção e as intervenções mais adequadas $^{18-21}$.

Entretanto, a crescente necessidade de atendimento às vítimas de violência que chegam aos serviços de saúde, que vem ocorrendo a partir de 1980 no Brasil ${ }^{22,23}$, evidencia as debilidades estruturais e organizacionais do sistema de saúde e as dificuldades de seus profissionais no atendimento e acompanhamento dessas pessoas. Em última análise, esta situação tem contribuído para a reincidência e agravamento de casos, pela falta de implementação de medidas preventivas e de intervenções apropriadas. Certamente, muitos desses problemas estão relacionados ao processo de formação e capacitação dos membros das equipes de saúde.

Sabe-se que a formação profissional no campo da violência é precária. De acordo com Bourroul ${ }^{24}$, poucos profissionais reconhecem seu papel no tratamento, afirmando que não estão preparados para lidar com vítimas de violência. Esse autor afirma que questões específicas do próprio profissional, entre elas o medo, devem ser destacadas na formação, para que ele possa ser preparado para desempenhar melhor o seu papel.

Uma formação socialmente contextualizada, que promova uma articulação estreita entre o mundo do ensino, do trabalho e a realidade, poderá instrumentalizar os profissionais para o enfrentamento de problemas e das reais necessidades de saúde da população ${ }^{25,26}$.

O manual Violência intrafamiliar - orientações para a prática em serviço, elaborado pelo Ministério da Saúde, vem ao encontro dessas necessidades. Com o objetivo de apoiar os Estados e Municípios na implementação de ações que visem à promoção da igualdade e ao exercício dos direitos humanos, objetiva orientar profissionais nas áreas de diagnóstico, tratamento e prevenção da violência, estimulando o estreitamento das relações entre as instituições das áreas de saúde, segurança, educação e defesa dos direitos, entre outros ${ }^{4}$.

Entretanto, existe uma carência de avaliação desses materiais instrucionais, que são publicados e distribuídos sem que se desen- 
volvam metodologias para capacitar os profissionais para o seu uso adequado.

O presente trabalho é parte de uma pesquisa cujo objeto envolveu as diretrizes para a formação médica e de Enfermagem sobre violência intrafamiliar a partir de orientações da prática em serviço recomendadas pelo Ministério da Saúde. Seu principal objetivo foi investigar a inserção do tema violência intrafamiliar nos currículos dos cursos de Medicina e de Enfermagem e a percepção de professores que poderiam favorecer a efetivação de tais propostas.

A etapa da pesquisa relatada neste artigo buscou identificar percepções, críticas e sugestões dos professores sobre o manual técnico Violência intrafamiliar: orientações para a prática em serviço. Embora esta ferramenta tenha sido dirigida aos profissionais que atuam nos serviços da atenção básica em saúde do SUS, foi adotada nesta pesquisa por se tratar de orientação oficial que aborda especificamente a violência intrafamiliar. Ao se realizar esta análise avaliativa, busca-se contribuir para aperfeiçoar os materiais instrucionais sobre o tema.

\section{METODOLOGIA}

A partir de uma perspectiva interinstitucional e interdisciplinar, foi realizado estudo de cunho exploratório que se pautou nos princípios da triangulação de métodos, conjugando técnica quantitativa com qualitativa, buscando constituir uma "rede de olhares" a partir de reflexões e debates de pesquisadores de formações variadas. taxas de mortalidade por causas externas acima da média nacional e equipe de pesquisadores que lidam com o tema.

A pesquisa abrangeu todos os cursos de Enfermagem e Medicina de Cuiabá que existiam em 2005; já no Rio de Janeiro, foi selecionada uma amostra de conveniência das instituições de ensino. Desta forma, a amostra final ficou constituída por cinco faculdades de Enfermagem e cinco de Medicina (duas estaduais, duas federais e duas particulares no Município do Rio de Janeiro; duas federais e duas particulares no Município de Cuiabá).

Por medidas operacionais e de custos, decidiu-se que responderiam ao questionário de avaliação do manual apenas os coordenadores das disciplinas que uma análise prévia dos currículos identificou que abordavam a violência ou temas correlatos. A Tabela 1 apresenta o número de coordenadores de disciplinas incluídos na amostra proposta e o número dos que efetivamente responderam, segundo universidades e cursos.

Dos 99 coordenadores a serem incluídos no estudo, apenas 67 responderam o instrumento de pesquisa, com a seguinte distribuição: 26 professores dos cursos de Medicina e 41 dos cursos de Enfermagem; 49 pertenciam a cursos do Rio de Janeiro e 18 a Cuiabá; e, finalmente, 48 foram respondidos por docentes das instituições públicas e 19 pelos das universidades particulares.

O maior número de docentes respondentes que o de propostos observado na Faculdade de Enfermagem do Rio de Janeiro deve-se ao fato de o curso estar passando por reformulação à época da pesquisa e também pelo interesse de vários coordenadores em participar do estudo.

Tabela 1

Distribuição do número de coordenadores de disciplinas propostos na amostra e de respondentes, segundo universidades e cursos

\begin{tabular}{|c|c|c|c|c|c|c|c|c|c|c|}
\hline \multirow{3}{*}{ Cursos } & \multicolumn{10}{|c|}{ Universidades } \\
\hline & \multicolumn{2}{|c|}{$\begin{array}{c}\text { Federal } \\
\text { Rio }\end{array}$} & \multicolumn{2}{|c|}{$\begin{array}{c}\text { Estadual } \\
\text { Rio }\end{array}$} & \multicolumn{2}{|c|}{$\begin{array}{l}\text { Particular } \\
\quad \text { Rio }\end{array}$} & \multicolumn{2}{|c|}{$\begin{array}{l}\text { Federal } \\
\text { Cuiabá }\end{array}$} & \multicolumn{2}{|c|}{$\begin{array}{c}\text { Particular } \\
\text { Cuiabá }\end{array}$} \\
\hline & $\mathrm{P}^{(1)}$ & $\mathrm{R}^{(2)}$ & $\mathrm{P}$ & $\mathrm{R}$ & $\mathrm{P}$ & $\mathrm{R}$ & $\mathrm{P}$ & $\mathrm{R}$ & $\mathrm{P}$ & $\mathrm{R}$ \\
\hline Medicina & 14 & 10 & 11 & 5 & 14 & 4 & 8 & 4 & 11 & 3 \\
\hline Enfermagem & 4 & 13 & 9 & 9 & 12 & 8 & 8 & 7 & 8 & 4 \\
\hline
\end{tabular}

(1) Proposto; (2) Respondido.

Foram selecionadas as cidades do Rio de Janeiro e Cuiabá, por representarem, respectivamente, cidades de grande e médio porte, estarem situadas em diferentes regiões do País, possuírem
O questionário continha questões abertas e fechadas sobre as características e aplicabilidade do manual (conhecimento e aceitação de uso em sala de aula, alcance dos objetivos propostos, 
linguagem, conteúdo, sugestões para sua melhoria e inclusão de novos temas).

Foi proposto que cada coordenador fizesse a leitura apenas de partes do manual identificadas como tema afim à sua disciplina, sendo construídas duas matrizes temáticas para a análise uma para as faculdades de Medicina e outra para as de Enfermagem -, definindo quais capítulos do manual deveriam ser lidos pelos docentes de cada área do conhecimento. Todos foram orientados para ler a parte mais geral e introdutória do manual. Buscou-se, dessa forma, garantir ao mesmo tempo uma abordagem que contemplasse a diversidade das áreas de conhecimento e das disciplinas, a afinidade desses coordenadores com os temas a serem lidos e uma leitura não muito exaustiva, a fim de conseguir a adesão dos docentes para o estudo.

Os dados foram comparados em função do curso (Enfermagem ou Medicina), do Município pesquisado (Rio de Janeiro e Cuiabá) e da natureza administrativa da faculdade (pública ou particular). Na análise dos questionários, as informações foram processadas em um banco de dados no programa EpiData, versão 3.1. Na análise dos dados, foi utilizado o programa SPSS, versão 13.0, no qual se realizaram as frequências simples e relativas e as análises uni e bivariadas das variáveis de interesse.

A fim de verificar diferenças estatisticamente significativas, foi utilizado o teste exato de Fisher. O teste qui-quadrado foi aplicado apenas nos casos em que não havia frequências esperadas menores que cinco. O nível de significância adotado foi de $5 \%$ e $10 \%$.
A pesquisa foi aprovada pelo Comitê de Ética da ENSP / Fiocruz, os diretores das faculdades envolvidas assinaram autorização para permitir a coleta de dados em suas unidades, e os docentes assinaram o termo de consentimento livre e esclarecido, conforme preconiza a Resolução 196/96 do Conselho Nacional de Saúde, que regulamenta pesquisas com seres humanos.

\section{RESULTADOS E DISCUSSÃO}

Para vários docentes, a pesquisa possibilitou que tomassem conhecimento, tivessem acesso ao manual e realizassem sua leitura. O fato de poderem ficar com um exemplar foi motivo de satisfação.

Indagados sobre o conhecimento do manual do Ministério da Saúde, todos os profissionais de Cuiabá responderam que já haviam tido contato com ele, enquanto a grande maioria dos docentes do Rio de Janeiro (77,8\% de Medicina e 80,8\% de Enfermagem) não o conhecia.

Caberia investigar melhor o porquê dessa divergência. Algumas hipóteses podem ser levantadas: o fato de o manual ter sido mais bem divulgado em Cuiabá do que no Rio; o fato de os docentes de Cuiabá estarem mais inseridos na atenção básica; ou, ainda, estarem mais engajados nas discussões em torno do tema.

OGráfico 1 apresenta as opiniões dos docentes sobre as características do manual. A linguagem, em geral, foi considerada clara por todos os respondentes; a objetividade da linguagem foi mais bem avaliada pelos profissionais de Cuiabá, e os enfermeiros tenderam a considerar, mais que os médicos, que a linguagem usada é sintética.

\section{Gráfico 1}

Avaliação dos docentes sobre a linguagem do manual, segundo cidade e curso.

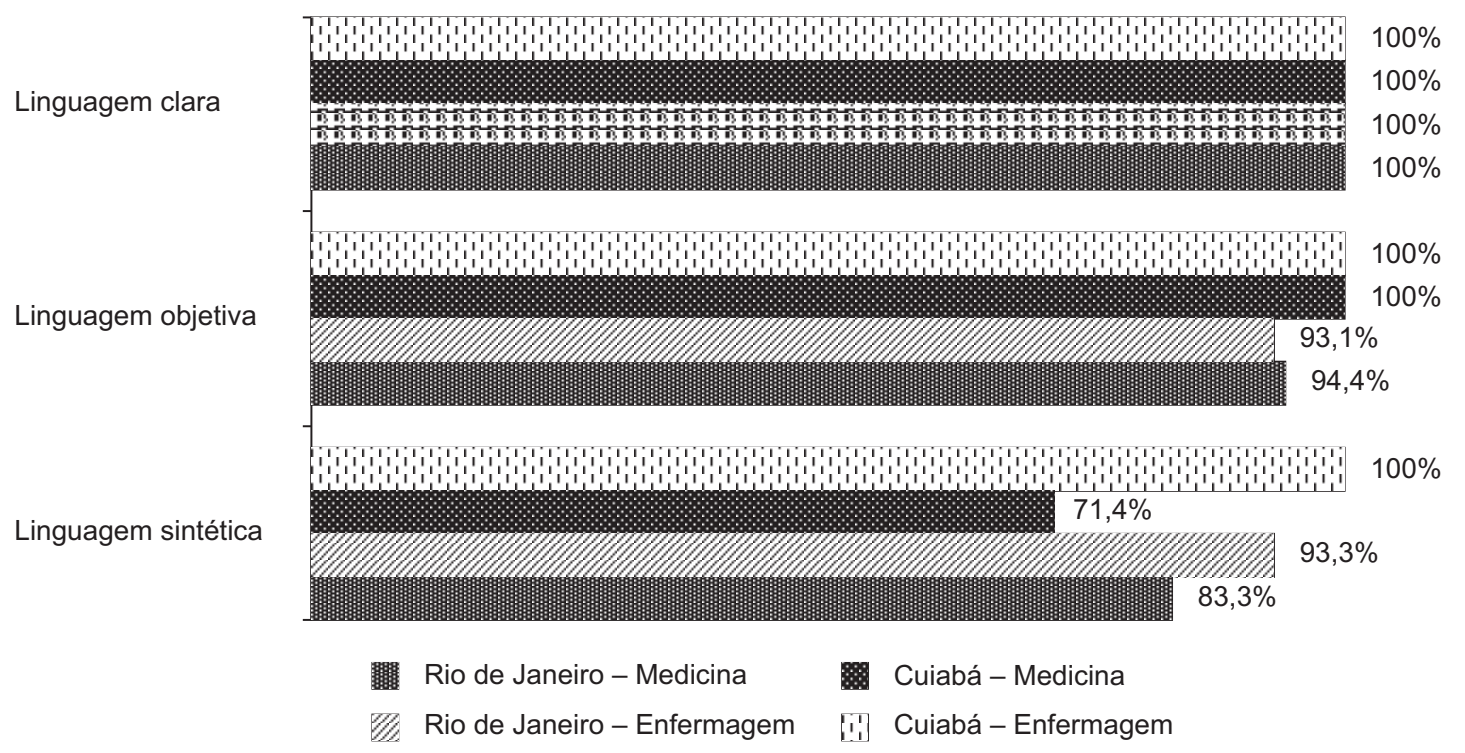

$33233(3): 329-338: 2009$ 
Na opinião dos docentes, há necessidade de acrescentar ilustrações ao manual. Recomendaram também que ele tivesse um formato menor e que fosse mais simples e objetivo.

No Gráfico 2 encontram-se as respostas dadas pelos professores em relação ao conteúdo do nanual: apesar de acharem o conteúdo adequado, este foi considerado insuficiente por boa parte dos professores das duas cidades, sobretudo em Cuiabá.

Gráfico 2
Em relação às sugestões sobre como melhorar o manual, os docentes reafirmaram que o conteúdo é insuficiente e sugeriram seu aprofundamento em geral e particularmente sobre o abuso sexual na infância, além da inserção de outros tipos de abuso.

A solicitação de aprofundamento sobre abuso sexual contra crianças é compatível com o que se observa na prática: este tema mobiliza muito os profissionais de saúde, que, em geral, não sabem en-

Avaliação dos docentes sobre o conteúdo do Manual, segundo cidade e curso.

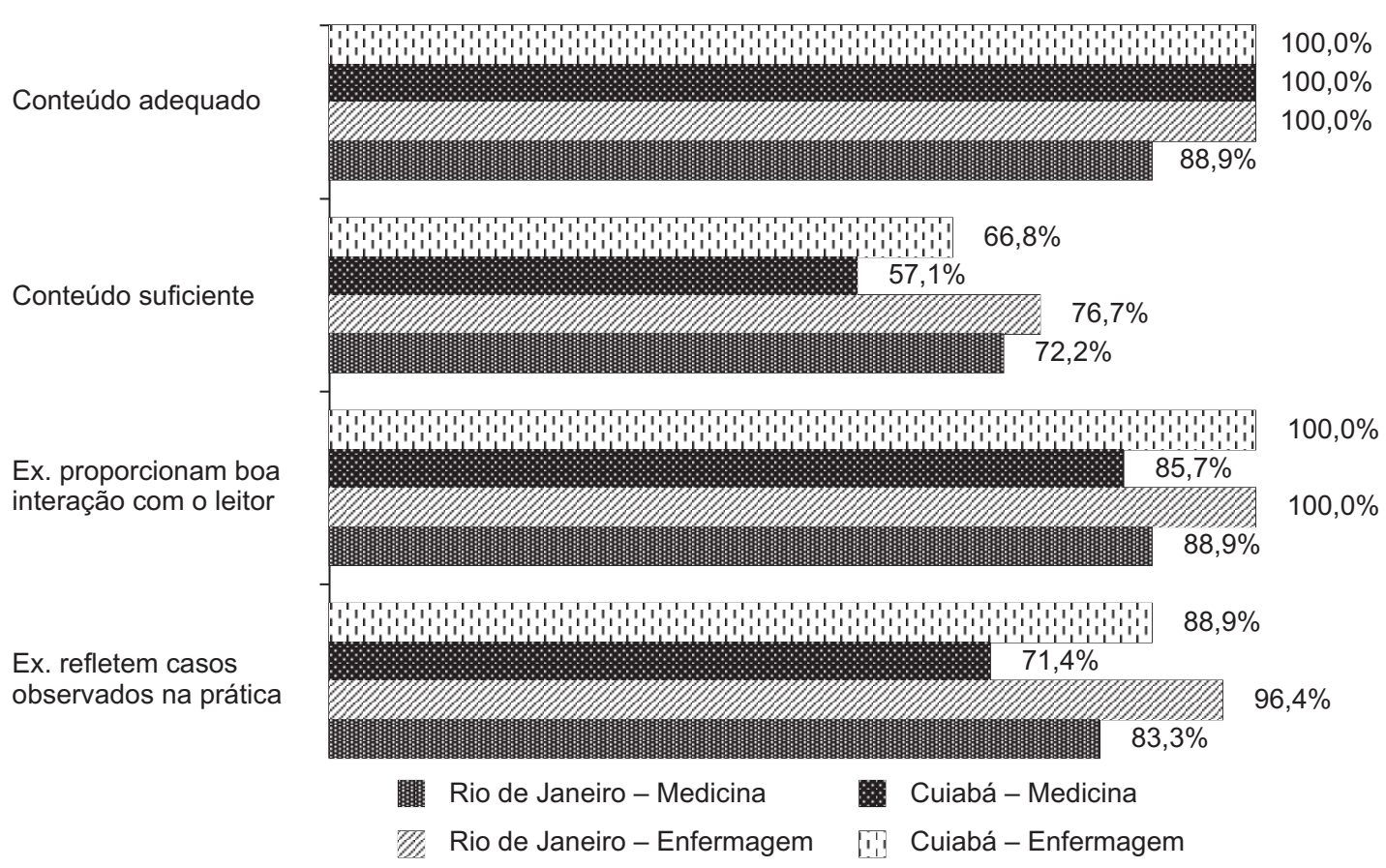

Quanto à interação dos exemplos com o leitor e ao fato de refletirem os casos atendidos na prática, os médicos das duas cidades foram mais críticos que os enfermeiros, embora a maioria das opiniões tenha sido favorável. É possível que os médicos, por atenderem situações clínicas mais complexas, não tenham se sentido contemplados por um manual que tem por objetivo atender a várias categorias profissionais na atenção básica e, consequentemente, não ter o compromisso de relatar questões clínicas e de tratamento mais específicas da área médica.

Na opinião dos docentes, o manual deveria incluir mais casos clínicos compatíveis com a realidade, com a abordagem de situações que suscitariam dúvidas, casos de difícil percepção e atendimentos sob condições "não ideais". Eles chamaram a atenção para o fato de o manual só exemplificar o abuso sexual na parte relativa à infância. Sugeriram ainda que o instrumento apresentasse as "síndromes mais comuns na infância", referindo-se provavelmente às síndromes de Munchausen by proxys, do BebêSacudido e da Criança Espancada, citadas superficialmente no material. frentar as situações e necessitam, inclusive, de suporte emocional para fazê-lo ${ }^{27-30}$.

Em relação à aplicabilidade do manual (Gráfico 3), os docentes foram mais favoráveis aos objetivos de ajudar na formação e sensibilização dos profissionais de saúde para o atendimento de pessoas em situação de violência. No entanto, avaliaram com restrições os objetivos mais amplos do manual, como, por exemplo, a capacidade do instrumento para capacitar profissionais de saúde para esse atendimento e estimular a atuação interinstitucional.

De fato, dificilmente um manual teria o poder de "capacitar" profissionais para a atenção às vítimas de violência sem estar associado a um treinamento prático ${ }^{31,32}$.

Analisando-se as respostas dos professores das faculdades do Rio, percebe-se que os que atuam no curso de Enfermagem foram mais críticos que os de Medicina em quase todas as questões (Gráfico3). Em Cuiabá, os professores de Enfermagem avaliaram melhor o manual que os de Medicina na maioria das questões relativas à sua aplicabilidade. Considerando-se as opiniões dos docentes dos 


\section{Gráfico 3}

Distribuição proporcional de avaliação dos docentes sobre a aplicabilidade do manual, segundo cidade e curso.

\section{"Você avalia o que o manual é capaz de:"}

Sensibilizar o profissional de saúde para o atendimento a pessoas em situação de violência

\section{Capacitar o profissional de saúde para o atendimento a pessoas em situação de violência}

Orientar o profissional de saúde nas ações de diagnóstico tratamento e prevenção da violência

Ajudar na formação do profissional de saúde para a prática em serviço no SUS

Fornecer condições para o profissional de saúde operacionalizar medidas de controle e prevenção da violência intrafamiliar

Fortalecer e potencializar ações e serviços para nova atitude, compromisso e colaboração em relação à violência intrafamiliar

Estimular o estreitamento das relações entre instituições atuantes nas áreas de saúde, garantia de direitos e movimentos sociais

Apoiar estados e municípios na implementação de ações que promovam igualdade e exercício dos direitos humanos

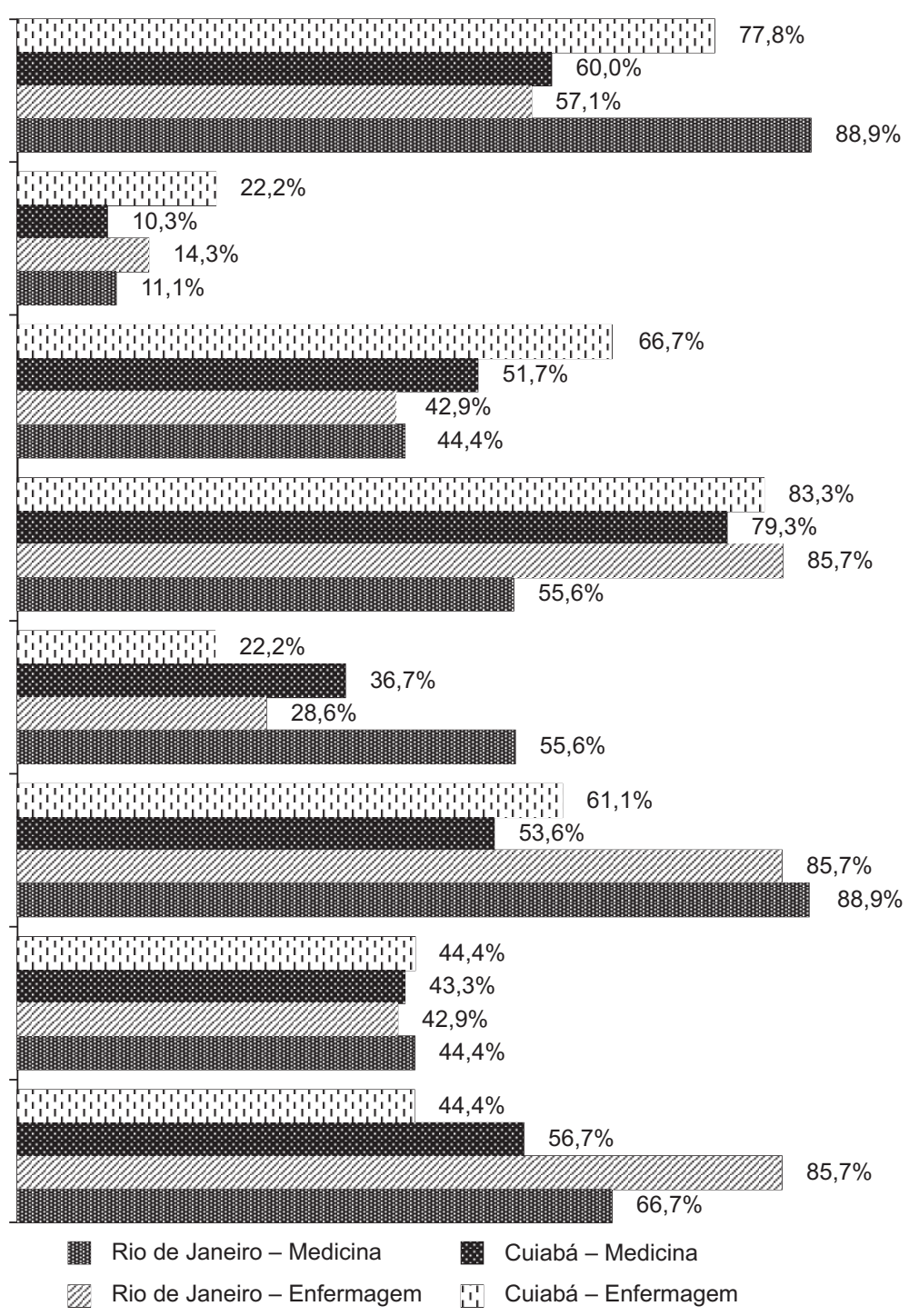

Apesar da boa avaliação do manual em termos de características e aplicabilidade e do conhecimento que afirmaram ter do mesmo, a grande maioria dos docentes não usa esse instrumento em suas aulas. Nenhum professor do curso de Medicina de Cuiabá informou tê-lo usado nas aulas, como pode ser visto no Gráfico 4.

Apesar de não adotá-lo como recurso didático, grande parte desses profissionais revelou que poderia fazê-lo. Essa adesão foi menor entre os entrevistados do Rio de Janeiro. A diferença observada entre os cursos de Enfermagem e Medicina do Rio de Janeiro foi estatisticamente significativa, com os enfermeiros sendo bem mais favoráveis à adesão ao manual $(\mathrm{p}=0,003)$. $=0,084)$. 


\section{Gráfico 4}

Distribuição proporcional das opiniões dos docentes quanto à aplicação didática do Manual, segundo cidade e curso.

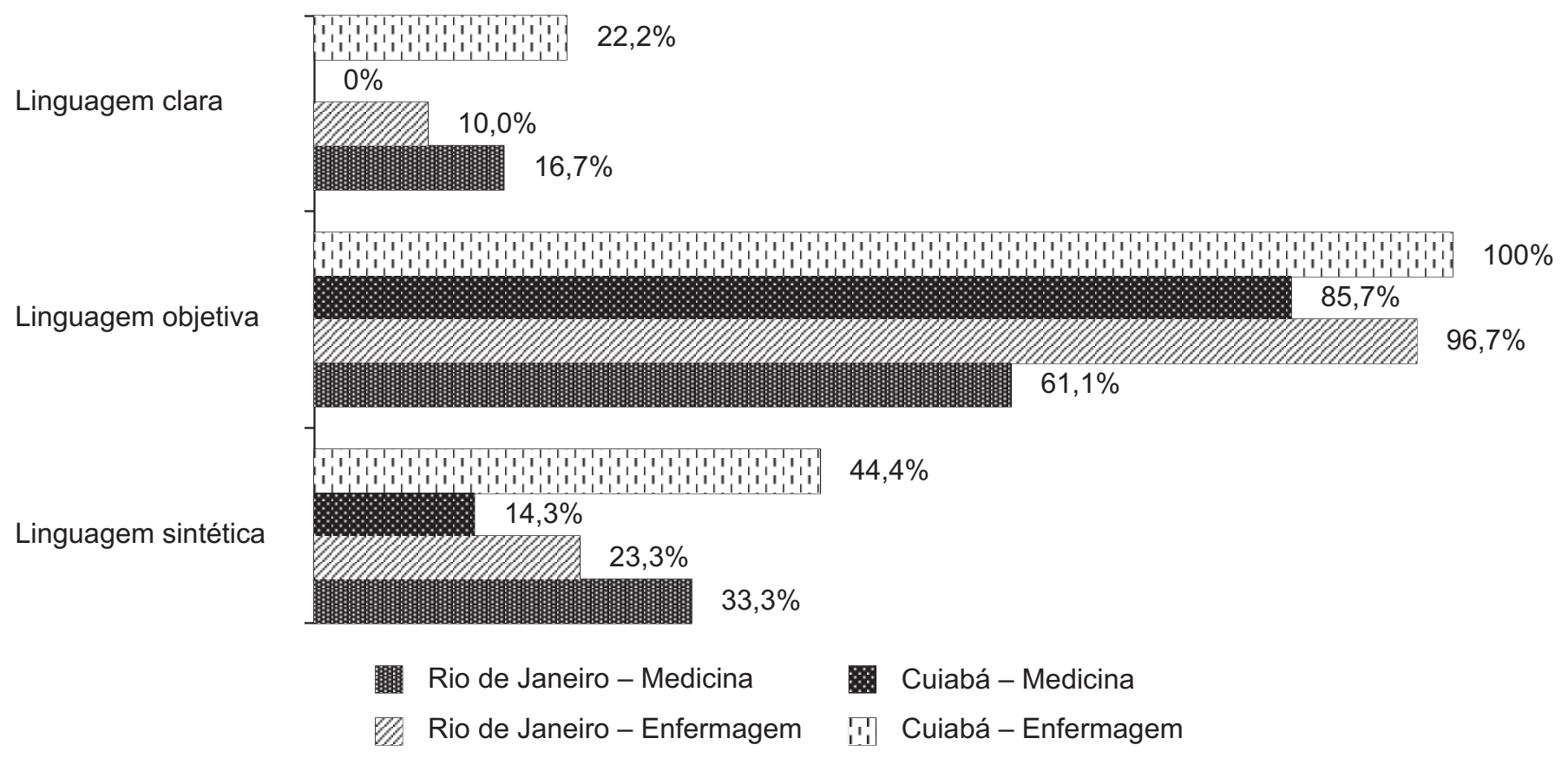

Ainda de acordo com o Gráfico 4 e de forma complementar às opiniões sobre a aplicabilidade do manual, observamos que os professores de Medicina de Cuiabá e de Enfermagem do Rio julgaram que o manual, por si só, não seria suficiente para cumprir a tarefa de formar os alunos no tema da violência intrafamiliar.

Vale lembrar que todos os professores de Cuiabá afirmaram conhecer o manual, mas apenas $16,7 \%$ dos enfermeiros fizeram uso desse material em suas disciplinas. Os professores da Enfermagem do Rio de Janeiro afirmaram que conhecem o manual $(80,8 \%)$, avaliaram positivamente seu conteúdo $(57,1 \%)$, mas, apesar disso, não o usavam em suas aulas. É possível que, como o conteúdo não tenha sido considerado suficiente, lancem mão de outras fontes.

Pesquisa realizada por Penna ${ }^{33}$ evidenciou que situações de pacientes vítimas de violência surgem frequentemente nos estágios. Entretanto, os professores do curso de Enfermagem pesquisado pela autora ainda realizavam uma abordagem informal da temática com seus alunos, sem sistematizá-la nos conhecimentos e instrumentais existentes, preferindo basear-se nas experiências empíricas dos docentes e discentes. Aliás, essa forma de abordagem do tema é extremamente frequente, tendo em vista a mobilização que o tema provoca e já que todos têm algo a di- zer com base em suas experiências, sem se preocupar em fundamentar o pensamento para além do senso comum.

Foi solicitado aos docentes que priorizassem em ordem de importância outros temas não contemplados no manual, mas sugeridos pela pesquisa. Os temas "família como unidade de intervenção" e "Estatuto da Criança e do Adolescente" foram os mais escolhidos como prioritários para serem acrescentados ao manual. Em seguida, optaram pelos temas "direitos da família", "notificação dos casos" e "rede de atendimento". Por fim, identificaram o "atendimento ao agressor" e a "violência contra os homens". Os temas referentes à família como unidade de intervenção e às redes de atenção para atendimento e prevenção da violência também foram reforçados como importantes pelos docentes nas perguntas abertas.

É interessante perceber que os itens priorizados destacaram a atuação junto à família, revelando sintonia com as diretrizes de integralidade do SUS, e mostraram a existência de sensibilização e conscientização para o tema da violência contra a criança e o adolescente, pioneiro na agenda da saúde.

Por outro lado, a violência contra os homens e o atendimento ao agressor, temas bastante recentes nos fóruns e debates do setor saúde, apareceram nos últimos postos de prioridade para os docentes pesquisados. $\operatorname{Cesca}^{34}$ constata que há predomínio da 
perspectiva punitiva - e não de tratamento - quando se analisa a condição do agressor. Por conseguinte, se atribui pouca importância à prevenção e à qualidade de vida dos envolvidos em situação de violência intrafamiliar.

Sabe-se que os homens sofrem condições mais severas e crônicas de saúde do que as mulheres e representam percentual expressivo nas taxas de morbimortalidade (inclusive por violência), mas, apesar disto, frequentam pouco os serviços de atenção primária à saúde ${ }^{35}$. A baixa frequência dos homens nos níveis primários de atenção, onde poderiam ser discutidos aspectos da promoção da paz e prevenção da violência, associada ao fato de representarem grande parte dos agressores na violência intrafamiliar ${ }^{36-38}$ e aparecerem pouco como vítimas deste tipo de violência, é um aspecto que pode ter contribuído para a pouca valorização dos docentes em relação ao tema da violência sofrida por esta parcela da população.

Em relação ao atendimento ao agressor, como não estava especificado no questionário o tipo de agressão cometida, o docente pode ter interpretado que seria um tema muito especializado e, portanto, não ser considerado objeto de sua prática assistencial e de ensino. No entanto, é provável que a resposta fosse diferente se a pesquisa tivesse perguntado sobre a inclusão de temas como "ensinar práticas disciplinares não violentas", que pode ser uma forma de atendimento a quem comete abuso físico contra crianças. Isto pode ser visto através de alguns temas sugeridos pelos docentes nas perguntas abertas e que, de certa forma, buscaram dar subsídios para a compreensão e contextualização da violência, etapa considerada fundamental para uma atenção adequada ao agressor: "causas da violência", "enfoque psicológico da agressividade e violência", "contexto socioeconômico-cultural", "violência decorrente do uso/abuso de drogas", "responsabilização social e individual".

Outros temas indicados nas respostas abertas, alguns dos quais extrapolaram o âmbito familiar, foram: reflexos da violência intrafamiliar na vida do trabalhador ou no trabalho; protocolos clínicos de atendimento da violência, violência contra grupos minoritários (como homossexuais e portadores de HIV); questões raciais, culturais, éticas e religiosas; legislação brasileira relacionada ao tema; altas hospitalares prematuras para liberar leitos como forma de violência institucional; abuso sexual cometido por mulheres; pedofilia virtual; acolhimento de vítimas por meio de grupos de recepção com participação de especialistas; promoção de cultura de paz.

Foi também recomendada a inclusão de informações de serviços tais como telefones, endereços e formas de acesso a órgãos que realizam atendimento às vítimas e recebem denúncias (Conselhos Tutelares, Delegacias Especiais de Atendimento à Mu- lher). No entanto, é bom lembrar que o manual é um instrumento a ser usado em nível nacional, e isto dificulta a inclusão de listagens de serviços locais para o País inteiro.

É importante observar que, de modo geral, os docentes parecem não ter se sentido suficientemente informados a respeito de condutas diante de casos. Por isso sugeriram que o manual contivesse orientações sobre o acompanhamento dos casos, esquemas com operacionalização/fluxo de ações (protocolos de atendimento) e orientações sobre notificação. Consideraram que é importante esclarecer sobre o trabalho intersetorial (setor saúde, Conselho Tutelar, Delegacia de Polícia, etc.).

Por fim, contribuíram com sugestões quanto à divulgação e utilização do manual. Nesse sentido, opinaram que o material deveria ser de mais fácil acesso, disponibilizado na internet e numa versão mais resumida, a ser distribuída para comunidades, escolas, associações, Programa de Saúde da Família, entre outros. Também relataram seu desejo de que o manual fosse distribuído aos docentes, a fim de que pudessem trabalhá-lo junto com os alunos.

\section{CONSIDERAÇÕES FINAIS}

As políticas públicas, de modo geral, e as específicas de atenção e prevenção dos acidentes e violências, dirigidas à criança e ao adolescente, à mulher e ao idoso enfatizam o importante papel da inserção do tema no âmbito do ensino nos diversos níveis, devido à sua magnitude e impacto na saúde da população brasileira. Às políticas agregam-se leis como os Estatutos da Criança e do Adolescente e o do Idoso, que destacam que a violência precisa ser enfrentada. Uma forma de fazê-lo é sensibilizar, conscientizar, capacitar e formar pessoas dos mais diversos âmbitos da sociedade, mas, sobretudo, os alunos e profissionais do setor saúde, para onde convergem as vítimas desses eventos.

É preciso realçar que os estudantes necessitam ser incentivados a desenvolver a consciência das forças sociais maiores que afetam toda a vida e a saúde, e a reconhecer o seu papel potencial como cidadão, membro de uma comunidade que se posiciona contra a violência.

Para isto, é necessário desenvolver estratégias de ensino do tema que contemplem desde sua inserção nos currículos, passando pela formação dos próprios docentes, criando campos de estágio que permitam vivenciar a questão, até a elaboração de material didático que atenda as necessidades do ensino teórico e prático.

Pode-se concluir que, de modo geral, os docentes fizeram uma avaliação positiva do instrumento preconizado pelo Minis- 
tério da Saúde, embora reconhecessem que ele poderia ser melhorado em alguns aspectos.

O manual aqui estudado foi considerado insuficiente em vários aspectos da violência intrafamiliar, mas poderia ser útil para suscitar a discussão do tema de maneira mais genérica. Parece que o problema está no fato de que ele é muito abrangente e pouco específico. Ao mesmo tempo, o tema da violência é mais abordado nas disciplinas oferecidas nos períodos do meio dos cursos de Medicina e Enfermagem estudados ${ }^{39}$. Por isso, esse material instrucional acaba não sendo considerado suficiente para nenhum especialista que deseje aprofundar questões específicas de sua disciplina.

Sugere-se, pois, utilizar este material em disciplinas oferecidas no início dos cursos da área da saúde, com o objetivo de sensibilizar e conscientizar os graduandos, mostrando que a violência intrafamiliar ocorre em nossa população, afeta diversos segmentos da sociedade e é considerada um problema de saúde pública que traz consequências físicas e mentais. Estes alunos terão que lidar mais tarde com tais questões no desempenho de sua profissão e lançar mão de materiais como o aqui analisado, que apontam as principais formas de lidar com o problema da violência intrafamiliar ou outras formas de violência. Entretanto, é importante frisar que manuais como estes, por si só, não têm a pretensão de "capacitar" os alunos para o atendimento. No decorrer dos cursos, durante as disciplinas clínicas específicas e no treinamento prático, poderiam ser utilizados materiais com conteúdos mais aprofundados e voltados para a atenção.

\section{REFERÊNCIAS}

1. Minayo MCS. The inclusion of violence in the health agenda: historical trajectory. Cien Saúde Colet. 2006;11(2):375-83.

2. Brasil. Ministério da Saúde. Portaria no 936/GM?? de 18 de maio de 2004. Dispõe sobre a estruturação da Rede Nacional de Prevenção da Violência e Promoção da Saúde e a Implantação e Implementação de Núcleos de Prevenção à Violência em Estados e Municípios. Diário Oficial da União, no 96. Brasília, 20 maio 2004; Seção 1.

3. Brasil. Ministério da Saúde. Secretaria de Vigilância em Saúde. Política nacional de promoção da saúde. Brasília: Ministério da Saúde; 2006. (Série B. Textos Básicos de Saúde.)

4. Brasil. Ministério da Saúde. Secretaria de Políticas da Saúde. Violência intrafamiliar: orientações para a prática em serviço. Brasília: Ministério da Saúde; 2002. (Caderno de Atenção Básica, 8). (Série A. Normas e Manuais técnicos, 131).

5. Brasil. Ministério da Saúde. Programa da Assistência Integral à Saúde da Mulher. Brasília: Ministério da Saúde; 1984.

6. Brasil. Ministério da Saúde. Secretaria de Atenção à Saúde. Departamento de Ações Programáticas Estratégicas. Polí- tica nacional de atenção integral à saúde da mulher: princípios e diretrizes. Brasília: Ministério da Saúde; 2004.

7. Brasil. Ministério da Saúde. Secretaria de Assistência à Saúde. Notificação de maus tratos contra criança e adolescentes pelos profissionais de saúde: um passo a mais na cidadania em saúde. Brasília: Ministério da Saúde; 2002. (Serie A. Normas e manuais técnicos, 107)

8. Brasil. Portaria no 2.528 de 18 de outubro de 2006. Política Nacional de Saúde da Pessoa Idosa. Diário Oficial da União. Brasília, 19 out. 2006.

9. Brasil. Senado Federal. 5 out. 1988. Constituição da República Federativa do Brasil. Brasília: Senado Federal; 1988.

10. Brasil. Lei no 7.853 de 24 de outubro de 1989. Apoio aos portadores de deficiência. Diário Oficial da União. Brasília, 25 out. 1989.

11. Brasil. Ministério da Saúde. Cartilha sobre violência contra criança e adolescente: proposta preliminar de prevenção e assistência à violência doméstica. Brasília: OPAS;OMS; 1997.

12. Brasil. Ministério da Saúde. Secretaria de Atenção à Saúde. Departamento de Ações Programáticas Estratégicas. Prevenção e tratamento dos agravos resultantes da violência sexual contra mulheres e adolescentes. Brasília; 1999. Norma técnica.

13. Brasil. Secretaria Especial de Políticas para as Mulheres. Programa de Prevenção, Assistência e Combate à Violência contra a Mulher - Plano Nacional: diálogos sobre violência doméstica e de gênero: construindo políticas públicas. Brasília: Secretaria Especial de Políticas para as Mulheres; 2003.

14. Brasil. Ministério da Saúde. Prevenção e tratamento dos agravos resultantes da violência sexual contra mulheres e adolescentes. 2a ed. Brasília; 2005. Norma técnica. (Série A. Normas e manuais técnicos). (Série Direitos sexuais e Direitos reprodutivos - Cadernos, 6).

15. Brasil. Ministério da Saúde. Secretaria de Atenção à Saúde. Departamento de Ações Programáticas Estratégicas. Atenção Integral para mulheres e adolescentes em situação de violência doméstica e sexual: matriz pedagógica para formação de redes. Brasília: Editora do Ministério da Saúde; 2006. Textos Básicos de Saúde, Série B.

16. Sociedade Brasileira de Pediatria; Fundação Oswaldo Cruz, Centro Latino Americano de Estudos de Violência e Saúde Jorge Careli e Escola Nacional de Saúde Pública Sergio Arouca. Guia de atuação frente a maus tratos na infância e adolescência: orientações para pediatras e demais profissionais que trabalham com crianças e adolescência. 2a ed. Rio de Janeiro: SBP; 2001.

17. Lima CA. Violência faz mal à saúde. Brasília: Ministério da Saúde; 2004. 
18. Rosenberg ML, Fenley MA, Johnson D, Short L. Bridging prevention and practice: public health and family violence. Acad Med. 1997;72(1 Supl):13-8.

19. Short LM, Cotton D, Hodgson CS. Evaluation of the module on domestic violence at the UCLA School of Medicine. Acad Med. 1997;72(1 Supl):75-92.

20. Weiss LB, Kripke EN, Coonse HL, O’Brien MK. Integrating a domestic violence education program into a medical school curriculum: challenges and strategies.Teache Learn Med. 2000;12(3):133-40.

21. Carney DM, McKibbin L. Screening for domestic violence. Nurs Manage. 2003;34(9):35-6.

22. Minayo MCS, Souza ER, Malaquias JV, Reis AC, Santos NC, Veiga JPC et al. Análise da morbidade hospitalar por lesões e envenenamentos no Brasil em 2000. In: Minayo MCS, Souza ER, (org.). Violência sob o olhar da saúde: a infrapolítica da contemporaneidade brasileira. Rio de Janeiro: Fiocruz; 2003. p.109-30.

23. Souza ER, Lima MLC. The panorama of urban violence in Brazil and its capitals. Cien Saude Colet. 2006;11(2):363-74.

24. Bourroul MLM. A violência doméstica contra a criança e o adolescente e o ensino de pediatria na residência médica. São Paulo; 2005. Mestrado [Dissertação] - Programa de Pós-Graduação em Ciências da Coordenadoria de Controle de Doenças da Secretaria de Estado da Saúde de São Paulo.

25. Costa HOG. A problematização da violência como experiência de ensinar em saúde. Interface Comun Saúde Educ. 1999;3(5):63-74.

26. March C, Koifman L, Pontes ALM, Saippa-Oliveira G, Silva Jr AGS, Fernandez VS. O currículo de medicina da Universidade Federal Fluminense: revisitando uma experiência. In: Pinheiro R, Ceccim RB, Mattos RA, (Orgs.). Ensinar saúde: a integralidade e o SUS nos cursos de graduação na área da saúde. $2^{\underline{a}}$ ed. Rio de Janeiro: IMS/UERJ; Cepesq; Abrasco; 2006. p.295-309.

27. Reid SA, Glasser M. Primary care physicians' recognition of and attitudes toward domestic violence. Acad Med 1997;72(1):51-3.

28. Araújo MF. Violência e abuso sexual na família.Psicol Estudo. 2002;7(2):3-11.

29. Azambuja MPR. Violência doméstica: reflexões sobre o agir profissional. Psicol Cienc Prof 2005;25(1):4-13.

30. Vidal A, Torres RR, Amador AA. Physicians and nurses: knowledge of clinical management of sexual aggression in children based on the sexual aggression survivors manual in Puerto Rico. P R Health Sci J. 2007;26(2):141-5.
31. Gongdon TW. A medical student's perspective on education about domestic violence. Acad Med. 1997;72(1Suppl):7-9.

32. Dickstein LJ. Practical recommendations for supporting medical students and faculty in learning about family violence.Acad Med 1997;72(1 Supl):105-9.

33. Penna LHG. A temática da violência contra a mulher na formação da enfermeira. Rio de Janeiro; 2005. Doutorado [Tese] - Instituto Fernandes Figueira, Fundação Oswaldo Cruz.

34. Cesca TB. O papel do psicólogo jurídico na violência intrafamiliar: possíveis articulações. Psicol Soc. 2004;16(3):41-6.

35. Gomes R, Nascimento EF, Araújo FC. Por que os homens buscam menos os serviços de saúde do que as mulheres? as explicações de homens com baixa escolaridade e homens com ensino superior. Cad Saúde Pública. 2007;23(3):565-74.

36. Schraiber LB, Gomes R, Couto MT. Homens e saúde na pauta da Saúde Coletiva. Ciênc Saúde Colet. 2005;10(1):7-17.

37. Marinheiro ALV, Vieira EM, Souza L. Prevalência da violência contra a mulher usuária de serviço de saúde. Rev Saúde Pública 2006;40(4):604-10.

38. Schraiber LB, D'Oliveira AFPL, Couto MT, Hanada H, Kiss LB, Durand JG et al. Violência contra mulheres entre usuárias de serviços públicos de saúde da Grande São Paulo. Rev Saúde Pública 2007;41(3):359-67.

39. Souza ER, Penna LHG, Ferreira AL, Tavares CMM, Santos NC. O tema violência intrafamiliar em currículos de graduação em Enfermagem e Medicina. Rev Enferm UERJ. 2008;16(1):13-9.

Organização de fomento: CNPq/DECIT- MS.

Número de cadastro do projeto: 505237/2004

\section{CONTRIBUIÇÃO DOS AUTORES}

Todas as autoras participaram na concepção, planejamento, execução, análise e interpretação dos dados, redação ou revisão do manuscrito de forma intelectualmente significativa.

\section{CONFLITO DE INTERESSES}

Declarou não haver

\section{ENDEREÇO PARA CORRESPONDÊNCIA}

Edinilsa Ramos de Souza

Avenida Brasil, 4036, sala 700

Manguinhos - Rio de Janeiro

CEP: 21040-061 RJ

E-mail: edinilsa@claves.fiocruz.br 